\title{
Bone marrow mesenchymal stem cells orthotopictransplantation reverse diabetes
}

\author{
Furong $\mathrm{Li}^{1}$, Caihong Chang ${ }^{2}$, Hanxin Zhou ${ }^{1}$, Feili Gong ${ }^{2}$ \\ ${ }^{I}$ Clinical medical research center, The Second Clinic Medical College (Shenzhen People's hospital), JiNan University, ShenZhen, \\ 518020, China; ' Immunology department of Tongji Medical college, Huazhong University of Science and Technology, WuHan \\ 430032, China
}

Mesenchymal stem cells(MSCs) transplantation to overcome deficient $\beta$-cell population is an attractive proposal. Nevertheless, the efficiency of adult stem cells to differentiate into islet-like cells in vitro is low at present. Stem cell differentiation is controlled by extra-cellular cues from the environment and by intrinsic genetic programs within the stem cell. The present study aimed to explore whether diabetic microenvironment could induce MSCs to trans-differentiate to islet cells and compensate insufficient $\beta$-cell. We transplanted male EGFP transduct MSCs to experimental diabetic female pig by multipoint injecting into subcapsular pancreas. MSCs not only act as "seed" cells, but also may exert a clinically useful immune modulatory effect, because of their immune privilege. Four weeks later, glucose level in serum began to decrease gradually, and was approximate to normal value. Reversal of diabetes was evidenced by normal insulin level in serum and normal intra-peritoneal glucose tolerance test. Pancreatic neo-genesis islets were verified by histology and morphometry. EGFP and insulin protein coexpression indicated that some of neo-genesis islet cells derived from transplanted MSCs and had normal function. Coexpression of Y-chromosome SRY gene and insulin mRNA, Pdx-1 mRNA, future clarified the results. This study shows that bone marrow derived MSCs could contribute functional islets in diabetic pancreatic microenvironment and no obvious immune rejections, which provide a theoretical and clinical applications of pancreas regeneration.

Keywords: Guizhou Minipigs, bone marrow, mesenchymal stem cells (MSCs), transplantation, experimental diabetes Cell Research (2008) 18:s69. doi: 10.1038/cr.2008.159; published online 4 August 2008 